\title{
A COUNTER EXAMPLE ON COMMON \\ PERIODIC POINTS OF FUNCTIONS
}

\author{
ALIASGHAR ALIKHANI-KOOPAEI \\ Mathematics Department \\ University of Isfahan \\ Isfahan, Iran
}

(Received July 19, 1996)

ABSTRACT. By a counter example we show that two continuous functions defined on a compact metric space satisfying a certain semi metric need not have a common periodic point.

KEY WORDS AND PHRASES: Fixed points, periodic points.

1992 AMS SUBJECT CLASSIFICATION CODES: 26A16, 47H10.

\section{INTRODUCTION}

In [1] we defined the notion of a semi-metric and used it in a contractive type inequality to obtain some results regarding common fixed points of two functions. We proved Theorem 1.1 and gave a counter example illustrating that we cannot replace the contractive coefficient $a$ with 1. However, it is natural to ask (see [2]) if it is possible to prove a version of Theorem 1.1 with (1.1) amended to read strict inequality, $a$ replaced by 1 , and with the additional requirement that $x \neq y$, for the situation in which the functions are defined on a compact metric space $X$. Theorem 1.2 provides a partial answer to this question. Here we show that in general we can not expect to prove such a result. We begin with Theorem 1.1 and Theorem 1.2 as well as some preliminaries from [1].

THEOREM 1.1. Let $f$ and $g$ be selfmaps of the unit interval and let $h: I \times I \rightarrow[0, \infty)$ br: a function having property $P_{1}$. Suppose $g$ is continuous on $I$ and $A$ is a nonempty closed $g-\imath$ nvariant subset of $F(f)$. If there exists a real number $a, 0 \leq a<1$ such that for all $x$ and $y$ in $F(f), f$ and $g$ satisfy the following inequality:

$$
\begin{gathered}
h(f x, f y) \leq a \cdot \max \{h(g x, g y), h(g x, f x), h(g y, f y), \\
h(g y, f x), h(f x, g y)\},
\end{gathered}
$$

then $f$ and $g$ have a unrque common fixed point. 
THEOREM 1.2. Suppose $f$ and $g$ are two selfmaps of a compact metric space $X$ with $g$ continuous, and let $h: X \times X \rightarrow[0, \infty)$ be a function having property $P_{1}$. If for all $x \neq y$ in $X . f$ and $g$ satisfy the following inequality:

$$
\begin{gathered}
h(f x, f y)<\max \{h(g x, g y), h(g x, f x), h(g y, f y), \\
h(g y, f x), h(f x, g y)\},
\end{gathered}
$$

then one of the following holds:

(2) either $f$ and $g$ have a common fixed point.

(2i) or every nonempty closed $g$-invariant subset of $F(f)$ contains a perfect minimal set $B$ such that the functions $\phi_{1}(x)=h(g x, x)$ and $\phi_{2}(x)=h(x, g x)$, do not attain their minimum or maximum on $B$.

Throughout $g^{n}$ denotes the $n$ fold composition of $g$ with itself and $X$ is a compact metric space. The orbit of $x$ under the homeomorphism $g$ ( a one to one function $g$ ), $O(g, x)$ is the set $\left\{g^{h}(x):-\infty<k<\infty\right\}$. A subset $Y$ of $X$ is called invariant under $g$ if $g(Y) \subseteq Y$. A closed, invariant, nonempty subset of $X$ is called minimal if it contains no proper subset that is also closed, invariant and nonempty. The sets $P(f)$ and $F(f)$ are the sets of periodic points and the fixed points of $f$, respectively. The space $\Sigma_{2}=\left\{s=\left(s_{0} s_{1} s_{2} \ldots\right): s_{j}=0\right.$ or 1$\}$ is called the sequence space on the two symbols 0 and 1 . For two sequences $s=\left(s_{0} s_{1} s_{2} \ldots\right)$ and $t=\left(t_{0} t_{1} t_{2} \ldots\right)$, their distance is defined by $d[s, t]=\sum_{t=0}^{\infty}\left|s_{\imath}-t_{\imath}\right| / 2^{2}$. It is clear that $\left(\Sigma_{2}, d\right)$ is a compact metric space.

Let $C$ be the Cantor Middle-Third set obtained as follows. Let $A_{0}=(1 / 3,2 / 3)$ be the middle third of the unit interval $I$ and $I_{0}=I-A_{0}$. Let $A_{1}=(1 / 9,2 / 9) \cup(7 / 9,8 / 9)$ be the middle third of the two intervals in $I_{0}$ and $I_{1}=I_{0}-A_{1}$. Inductively, let $A_{n}$ denote the middle third of the intervals in $I_{n-1}$ and let $I_{n}=I_{n-1}-A_{n}$ and $C=\cap_{n \geq 0} I_{n}$. For each $x \in C$, we attach an infinite sequence of $0^{\prime}$ s and $1^{\prime} \mathrm{s}, S(x)=\left(s_{0} s_{1} s_{2} \ldots\right)$, according to the rule: $s_{0}=1$ if $x$ belongs to the left component of $I_{0} ; s_{0}=0$ if $x$ belongs to the right component of $I_{0}$. Since $x$ belongs to some component of $I_{n-1}$, and $I_{n}$ is obtained by removing the middle third of this interval. Therefore we may set $s_{n}=1$ if $x$ belongs to the left hand interval and $s_{n}=0$ otherwise. By this way we can think of elements of the Cantor set $C$ as elements of $\Sigma_{2}$ and vice versa. Define $A: \Sigma_{2} \rightarrow \Sigma_{2}$ by $A\left(s_{0} s_{1} s_{2} \ldots\right)=\left(s_{0} s_{1} s_{2} \ldots\right)+(100 \ldots) \bmod 2$, i.e., $A$ is obtained by adding 1 mod 2 to $s_{0}$ and carrying the result to the right. For example $A(0000 \ldots)=(1000 \ldots), A(1000 \ldots)=$ $(0100 \ldots), A(0100 \ldots)=(1100 \ldots), A(110 \overline{110} \ldots)=(001 \overline{110} \ldots), A(111 \ldots)=(000 \ldots)$. The map $A$ is known as the adding machine (see [4]).

\section{RESULTS}

We first show that $A$ is a homeomorphism on $\Sigma_{2}$ ( or in another word $C$ ) and the orbit of every point of $C$ under $A$ is dense in $C=\Sigma_{2}$. Since $\Sigma_{2}$ does not have a nonempty proper closed invariant subset under $A$, it is a perfect minimal set. 
LEMMA 2.1 $A$ is a homeomorphism from $C$ to itself.

PROOF. To see this we show that $A$ is continuous, one one, onto on $C$ with $A^{-1}$ also continuous.

To see that $A$ is continuous, let $x$ be an arbitrary point of $C$ and $\epsilon>0$. Let $N$ be a positive integer such that $1 / 2^{N}<\epsilon$. Choose $\delta=1 / 2^{N}$. If $d(y, x)<\delta$, the sequences $x$ and $y$ have identical first $N$ elements, hence $A(x)$ and $A(y)$ have also identical first $N$ terms. Thus $d(A(x), A(y))<1 / 2^{N}<\epsilon$, implying the continuity of $A$ at $x$.

To see that $A$ is one one on $C$, let $x=\left(x_{0} x_{1} \ldots\right), y=\left(y_{0} y_{1} \ldots\right)$ be two points of $C$ with $x \neq y$, then there exists a least nonnegative integer $N$ such that $x_{N} \neq y_{N}$. Obviously the corresponding elements of the sequences $A(x)$ and $A(y)$ are different, hence $A$ is one one.

To see that $A$ is onto, let $y=\left(y_{0} y_{1} y_{2} \ldots\right)$ and $N$ be the smallest nonnegative integer such that $y_{N}=1$. Then for $x=\left(11 \ldots 0 y_{N+1} y_{N+2} \ldots\right)$ we have $A(x)=y$.

Since $\left(\Sigma_{2}, d\right)$ is a compact metric space and $A$ is continuous on $C=\Sigma_{2}$, the image of every closed subset of $C$ under $A$ is a closed set, implying the continuity of $A^{-1}$.

LEMMA 2.2. The orbit of every point of $C$ under $A$ is dense in $C$.

PROOF. Let $x=\left(x_{0} x_{1} x_{2} \ldots\right)$ and $y=\left(y_{0} y_{1} y_{2} \ldots\right)$ be two arbitrary points of $C=\Sigma_{2}$. For c $>0$, choose a positive integer $N$ so that $1 / 2^{N}<\epsilon$. Let $N_{1}$ be the least positive integer such that the sequences $x$ and $y$ have identical first $N_{1}-1$ elements. Then for $k_{1}=2^{N_{1}-1}$ the two sequences $A^{k_{1}}(x)$ and $y$ have at least $N_{1}$ identical first elements. Similarly suppose $N_{2}$ is the least positive integer such that the first $N_{2}-1$ elements of the two sequences $A^{k_{1}}(x)$ and $y$ are identical. Then $N_{2} \geq N_{1}+1$ and for $k_{2}=2^{N_{2}-1}$ the two sequences $A^{k_{1}+k_{2}}(x)$ and $y$ have identical first $N_{2}$ elements. By repeating this process we obtain a positive integer $m=k_{1}+k_{2}+\ldots+k_{l}$ such that $A^{m}(x)$ and $y$ have identical first $N$ elements, implying $d\left(A^{m}(x), y\right)<1 / 2^{N}<\epsilon$. Since $x$ and $y$ were arbitrary we may interchange the role of $x$ with $y$. Thus the result is established.

DEFINITION 2.1. Let $X$ be a compact metric space. The function $h: X \times X \rightarrow[0, \infty)$ is said to have property $P_{1}$ if it satisfies the following conditions:

(i): $h(x, y)=0$ if and only if $x=y$,

(ii): if $\lim _{n \rightarrow \infty} x_{n}=x_{0}, \lim _{n \rightarrow \infty} y_{n}=y_{0}$, and $\lim _{n \rightarrow \infty} h\left(x_{n}, y_{n}\right)=0$, then $x_{0}=y_{0}$.

The following theorem is based on an example which illustrates that assertion (ii) of Theorem 1.2 may occur.

THEOREM 2.1. There exist two continuous functions $f$ and $g$ selfmaps of a compact metric space $(X, d)$, a g-minimal perfect set $B \subseteq F(f)$ and a function $h: X \times X \rightarrow[0, \infty)$ having property $P_{1}$ such that for all $x \neq y$ both in $B, f$ and $g$ satisfy the following:

$$
\begin{gathered}
h(f x, f y)<\max \{h(g x, g y), h(g x, f x), h(g y, f y), \\
h(g y, f x), h(f x, g y)\},
\end{gathered}
$$

yet $f$ and $g$ do not have a common periodic point.

PROOF. Consider the compact metric space $\left(\Sigma_{2}, d\right)$. For each $x \in \Sigma_{2}$, let $f(x)=x$ and 
$g(x)=A(x)$, where $A$ is the adding machine. It is clear that $B=\Sigma_{2}$ is a $g$-invariant perfect subset of $F(f)$. Suppose $H=\{O(g, x): x \in B\}$. By the axiom of choice there is a set $E$ such that $E$ has exactly one element from each element of $H$. Choose an arbitrary point $x_{0} \in E$. Define the function $h: B \times B \rightarrow[0, \infty)$ as follows:

(a): $h(t, s)=0$ if $s=t$.

(b): Suppose $M=O\left(g, x_{0}\right) \times O\left(g, x_{0}\right)$. We define $h$ on $M$ as

(i): for $n, m \geq 0, n \neq m, h\left(g^{n} x_{0}, g^{m} x_{0}\right)=h\left(g^{m} x_{0}, g^{n} x_{0}\right)=7-1 / 2^{(m+n)}$.

(ii): For $m<0, n=0, h\left(g^{n} x_{0}, g^{m} x_{0}\right)=h\left(g^{m} x_{0}, g^{n} x_{0}\right)=3-1 / 2^{-m}$.

(iii): For $m<0<n, h\left(g^{n} x_{0}, g^{m} x_{0}\right)=h\left(g^{m} x_{0}, g^{n} x_{0}\right)=5-1 / 2^{(-m+n)}$.

(iv): For $m \neq n, m<0, n<0, h\left(g^{n} x_{0}, g^{m} x_{0}\right)=h\left(g^{m} x_{0}, g^{n} x_{0}\right)=1+1 / 2^{-(m+n)}$.

(c): Let $z \in E$ and $z \neq x_{0}$,

(i): for each integers $m$ and $n$, define $h\left(g^{n} z, g^{m} z\right)=h\left(g^{m} z, g^{n} z\right)=h\left(g^{n} x_{0}, g^{m} x_{0}\right)=h\left(g^{m} x_{0}, g^{n} x_{0}\right)$

(ii): For each integers $m$ and $n, \quad m \neq n$ and each $t \in E, z \in E, t \neq z$, define $h\left(g^{n} z, g^{m} t\right)=$ $h\left(g^{m} t, g^{n} z\right)=h\left(g^{n} x_{0}, g^{m} x_{0}\right)$.

(iii): For each integers $m \neq n$, define $h\left(g^{n} z, g^{m} x_{0}\right)=h\left(g^{m} x_{0}, g^{n} z\right)=h\left(g^{n} x_{0}, g^{m} x_{0}\right)$.

(iv): For each integer $n$ and each $t \neq z$ both different from $x_{0}$, define $h\left(g^{n}(z), g^{n}(t)\right)=h\left(g^{n}(t), g^{n}(z)\right)$ : $h\left(g^{n} z, g^{n} x_{0}\right)=h\left(g^{n} x_{0}, g^{n} z\right)= \begin{cases}4-1 / 2^{(n+2)} & 0 \leq n, \\ 3+1 / 2^{-n} & n<0 .\end{cases}$

Since $g$ is one to one on $B$, the function $h$ is well defined on $B \times B$. The function $h$ satisfies the property $P_{1}$ since, for $x=y, h(x, y)=0$ and for each $(x, y) \in B \times B, h(x, y) \geq 1$. It remains to show that for every $t \neq s$ in $B$ the inequality (2.1) is satisfied. To show this let $t, s \in B$, and $t \neq s$. We distinguish several different cases.

('ase 1: There exists $x_{0} \in E$ such that $t=g^{n}\left(x_{0}\right), s=g^{m}\left(x_{0}\right)$ for some integers $m$ and $n$.

(i): If $r n=n$ then $h(t, s)=0$, but $h(g t, s)>0$.

(ii) If $n, r n \geq 0$ and $m \neq n$, then $h(t, s)=h\left(g^{n} x_{0}, g^{m} x_{0}\right)=7-1 / 2^{(m+n)}$ and $h(g t, g s)=$ $h\left(g^{(n+1)} x_{0}, g^{(m+1)} x_{0}\right)=7-1 / 2^{(m+n+2)}$. Hence $h(t, s)<h(g t, g s)$.

(iii) If $m<0, n=0, h(t, s)=h\left(x_{0}, g^{m} x_{0}\right)=3-1 / 2^{-m}$. Suppose $m<-1$. Then $h(g t, g s)=$ $h\left(g x_{0}, g^{(m+1)} x_{0}\right)=5-1 / 2^{-m}$. If $m=-1$, then $h(t, s)=5 / 2$, but $h(g t, g s)=h\left(g x_{0}, g^{(m+1)} x_{0}\right)=$ $h\left(g x_{0}, x_{0}\right)=13 / 2$. Hence in this case we have $h(t, s)<h(g t, g s)$.

(iv) If $m<0<n$, then $h(t, s)=h\left(g^{n} x_{0}, g^{m} x_{0}\right)=5-1 / 2^{(-m+n)}$. If $m=-1$, we have $h(g t, g s)=h\left(g^{(n+1)} x_{0}, g^{(m+1)} x_{0}\right)=h\left(g^{(n+1)} x_{0}, x_{0}\right)=7-1 / 2^{(n+1)}$. If $m<-1$, then $h(g t, s)=$ $h\left(g^{(n+1)} x_{0}, g^{m} x_{0}\right)=5-1 / 2^{(-m+n+1)}$ and $h(g t, t)=h\left(g^{(n+1)} x_{0}, g^{n} x_{0}\right)=7-1 / 2^{(n+1+n)}=7-$ $1 / 2^{(2 n+1)}$. Hence we have $h(t, s)<\max \{h(g t, g s), h(g t, s)\}$ and $h(t, s)<\max \{h(g t, g s), h(g t, t)\}$.

(v) If $m \neq n, m<0, n<0$, then $h(t, s)=h\left(g^{n} x_{0}, g^{m} x_{0}\right)=1+1 / 2^{-(m+n)}$. Suppose either $m=-1$ or $n=-1$. Without loss of generality we may assume that $m=-1$. Then we have $h(t, s)=$ $1+1 / 2^{(1-n)}$ and $h(g t, g s)=h\left(g^{(n+1)} x_{0}, g^{(m+1)} x_{0}\right)=h\left(g^{(n+1)} x_{0}, x_{0}\right)=3-1 / 2^{-(n+1)}$. For $m<-1$ and $n<-1$ we also have $h(t, s)=1+1 / 2^{-(m+n)}$ and $h(g t, g s)=h\left(g^{(n+1)} x_{0}, g^{(m+1)} x_{0}\right)=1+$ $1 / 2^{-(m+n+2)}$, implying $h(t, s)<h(g t, g s)$. Thus again we have $h(t, s)<\max \{h(g t, g s), h(g t, s)\}$ 
and $h(t, s)<\max \{h(g t, g s), h(g t, t)\}$.

Case 2: There exist $x_{1} \in E, x_{2} \in E$ such that $t=g^{n}\left(x_{1}\right), s=g^{m}\left(x_{2}\right)$, for some integers $m$ and $n$. If $m \neq n$, then $h(t, s)=h\left(g^{n} x_{1}, g^{m} x_{2}\right)=h\left(g^{n} x_{0}, g^{m} x_{0}\right)<\max \{h(g t, g s), h(g t, s)\}$ and $h(t, s)<\max \{h(g t, g s), h(g t, t)\}$. If $n=m \geq 0$, then $h(t, s)=h\left(g^{n} x_{1}, g^{n} x_{2}\right)=4-1 / 2^{(n+2)}$ and $\mathrm{h}(\mathrm{gt}, \mathrm{gs})=\mathrm{h}\left(\mathrm{g}^{(n+1)} x_{1}, g^{(n+1)} x_{2}\right)=4-1 / 2^{(n+3)}$, thus $h(t, s)<h(g t, g s)$. If $n=m=-1$, then $h(t, s)=h\left(g^{-1} x_{1}, g^{-1} x_{2}\right)=7 / 2$, and $h(g t, g s)=h\left(x_{1}, x_{2}\right)=15 / 4$. If $n=m<-1$, then $h(t, s)=3+1 / 2^{-n}$ and $h(g t, g s)=3+1 / 2^{-(n+1)}$. Hence for this case we also have $h(t, s)<\max \{h(g t, g s), h(g t, s)\}$ and $h(t, s)<\max \{h(g t, g s), h(g t, t)\}$.

We see that inequality (2.1) is satisfied for every $t, s \in B$, yet $f$ and $g$ do not have any common periodic point. In fact the set of fixed points of $f$ is identical to $B$, while $g$ does not have any periodic point in $B$.

Remark 2.1. We may choose the functions $f$ and $g$ to be continuous selfmaps of the unit interval. For example let $\left\{\left(a_{n}, b_{n}\right)\right\}_{n=1}^{\infty}$ be the complementary intervals of the middle third Cantor set $C$. Define

$$
f(x)= \begin{cases}x & x \in C, \\ a_{n} & a_{n}<x \leq\left(a_{n}+b_{n}\right) / 2, \\ 2\left(x-b_{n}\right)+b_{n} & \left(a_{n}+b_{n}\right) / 2<x \leq b_{n},\end{cases}
$$

for $n=1,2,3, \ldots, g$ a continuous extension of $A$, and the semi metric $h$ on $C$ as in the above theorem. It is clear that $F(f)=C$ and the orbit of each point outside $C$ under $f\left(\right.$ c.e. $\left.\left\{x, f(x), f^{2}(x), f^{3}(x), \ldots\right\}\right)$ is attracted to a point in $C$, also $f$ and $g$ satisfy the inequality $(2.1)$ on $F(f)$, yet they do not have any common periodic points.

ACKNOWLEDGMENT. I would like to thank professor B.E. Rhoades for his comments (reference[3]) which led to this work. I Also wish to thank professor A. M. Bruckner for his valuable suggestions.

AUTHOR'S PRESENT ADDRESS. Department of Mathematics, The University of Tennessee at Chattanooga, Chattanooga, TN 37403- 2598.

\section{REFERENCES}

1. ALIKHANI-KOOPAEI, A. A., On common fixed and periodic points of commuting functions, International Journal of Mathematics and Mathematical Sciences, to appear.

2. RHOADES, B.E., A comparison of various definitions of contractive mappings, Trans. Amer. Math. Soc. 226(1977), 257-290.

3. RHOADES, B.E., Private Communication.

4. DEVANEY, R. L.,An introduction to chaotic dynamical systems, second edition, Addition-Wesley Publishing Inc., 1989. 


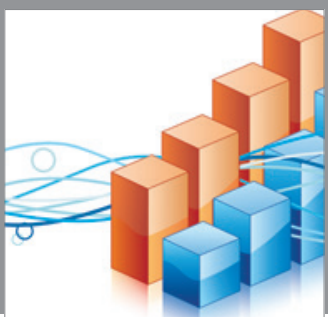

Advances in

Operations Research

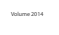

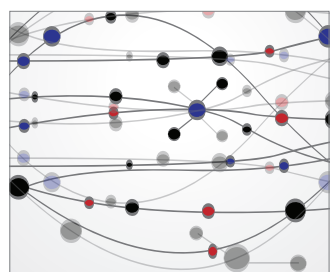

\section{The Scientific} World Journal
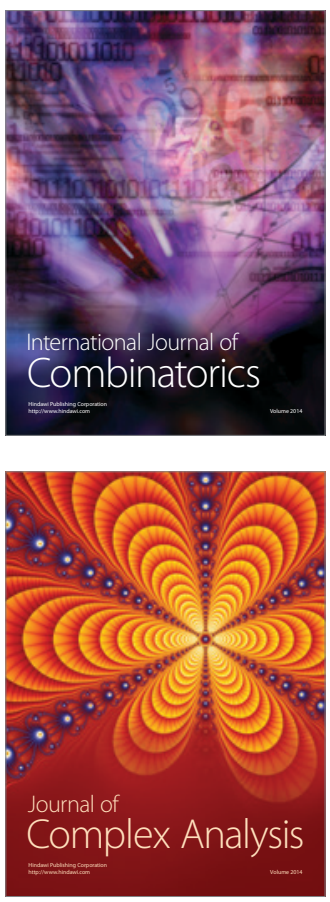

International Journal of

Mathematics and

Mathematical

Sciences
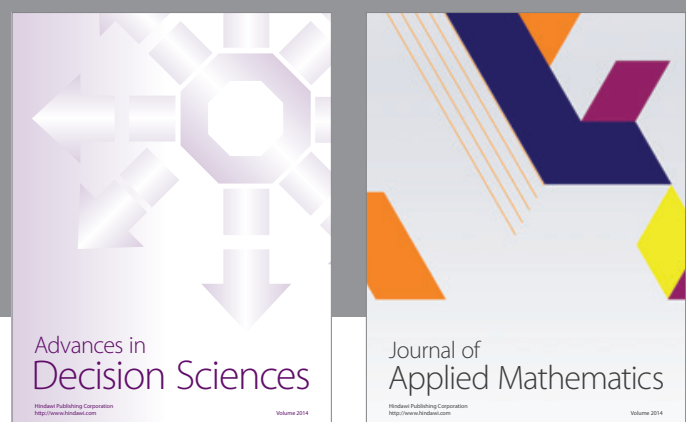

Journal of

Applied Mathematics
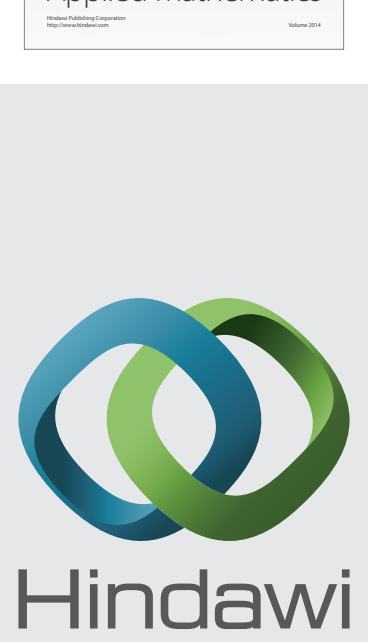

Submit your manuscripts at http://www.hindawi.com
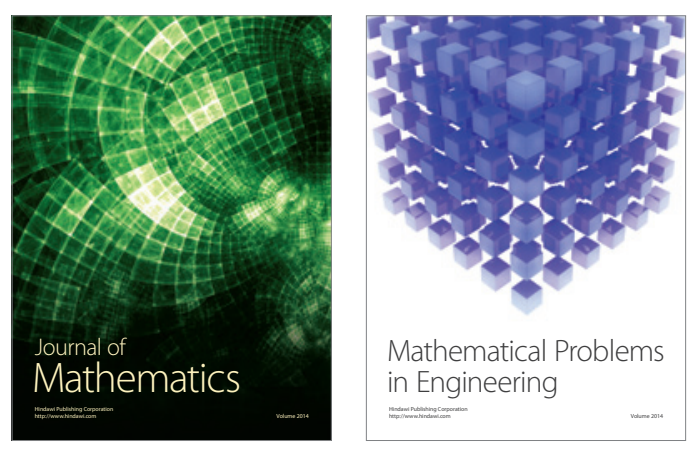

Mathematical Problems in Engineering
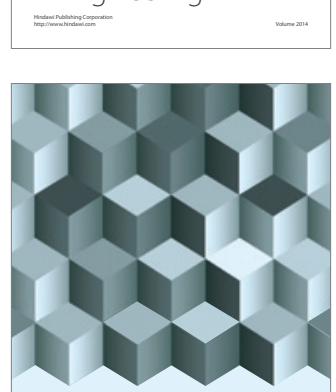

Journal of

Function Spaces
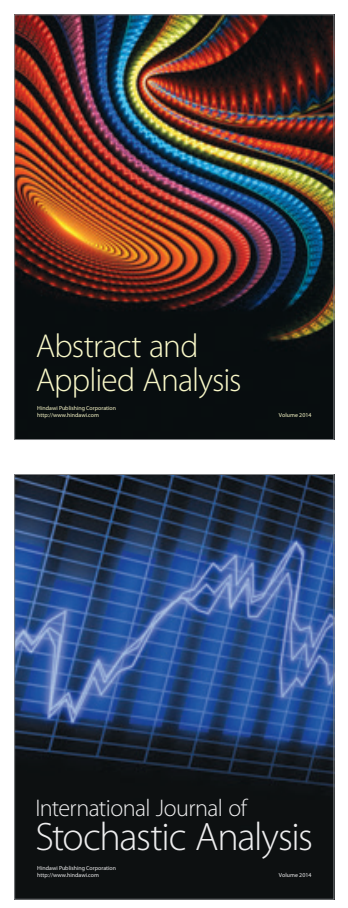

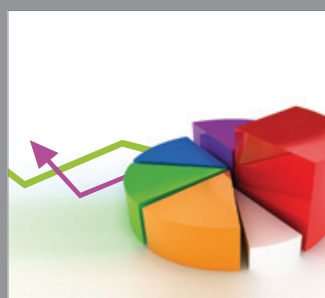

ournal of

Probability and Statistics

Promensencen
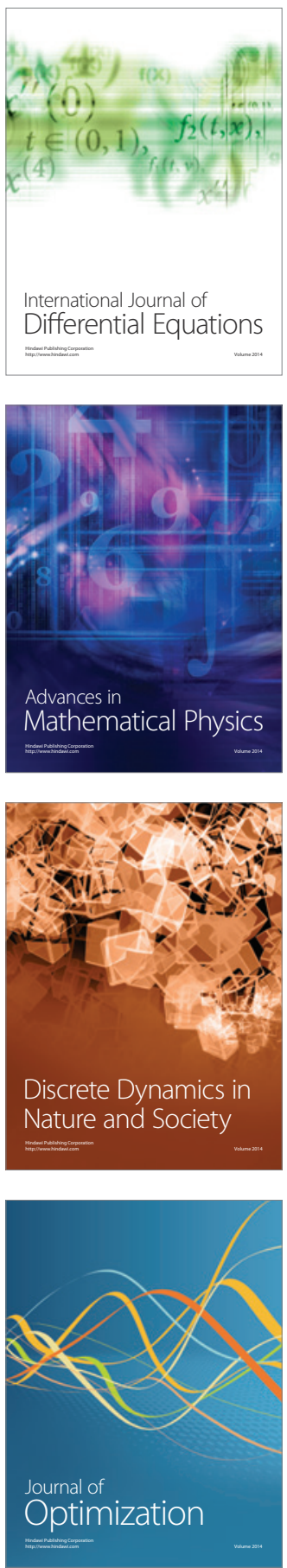